\title{
Navier-Stokes equations: an analysis of a possible gap to achieve the energy equality
}

\author{
Francesca Crispo* Carlo Romano Grisanti ${ }^{\dagger} \quad$ Paolo Maremonti ${ }^{\dagger}$
}

\begin{abstract}
The paper is concerned with the IBVP of the Navier-Stokes equations. The goal is the attempt to construct a weak solution enjoying an energy equality. This result is a natural continuation and improvement of the one obtained by the same authors in [2].
\end{abstract}

Keywords: Navier-Stokes equations, weak solutions, energy equality.

AMS Subject Classifications: 35Q30, 35B65, 76D05.

\section{Introduction}

This note concerns the 3D-Navier-Stokes initial boundary value problem:

$$
\begin{aligned}
& v_{t}+v \cdot \nabla v+\nabla \pi_{v}=\Delta v, \nabla \cdot v=0, \text { in }(0, T) \times \Omega, \\
& v=0 \text { on }(0, T) \times \partial \Omega, v(0, x)=v_{0}(x) \text { on }\{0\} \times \Omega .
\end{aligned}
$$

In system (1) $\Omega \subseteq \mathbb{R}^{3}$ is assumed to be bounded or exterior, and its boundary is smooth. The symbol $v$ denotes the kinetic field, $\pi_{v}$ is the pressure field, $v_{t}:=\frac{\partial}{\partial t} v$ and $v \cdot \nabla v:=v_{k} \frac{\partial}{\partial x_{k}} v$. We investigate on the existence of weak solutions. Here the notion of weak solution (see Definition 1) is meant in the sense of Leray's weak solution, but with no change in the arguments it is possible to consider a weak solution in the sense of Caffarelli-Kohn-Nirenberg [1]. In this connection we refer the interested reader to the paper [2] for details.

Definition 1. Let $v_{0} \in J^{2}(\Omega)$. A field $v:(0, \infty) \times \Omega \rightarrow \mathbb{R}^{3}$ is said a weak solution if the field $v$ verifies

i. for all $T>0, v \in L^{\infty}\left(0, T ; J^{2}(\Omega)\right) \cap L^{2}\left(0, T ; J^{1,2}(\Omega)\right)$,

ii. $\lim _{t \rightarrow 0}\left\|v(t)-v_{0}\right\|_{2}=0$,

*Dipartimento di Matematica e Fisica, Università degli Studi della Campania "L. Vanvitelli", via Vivaldi 43, 81100 Caserta, Italy. francesca.crispo@unicampania.it

${ }^{\dagger}$ Dipartimento di Matematica, Università di Pisa, via Buonarroti 1/c, 56127 Pisa, Italy. carlo.romano.grisanti@unipi.it

¥Dipartimento di Matematica e Fisica, Università degli Studi della Campania "L. Vanvitelli", via Vivaldi 43, 81100 Caserta, Italy. paolo.maremonti@unicampania.it

$\S$ The research activity of F. Crispo and P. Maremonti is performed under the auspices of GNFM-INdAM. The research activity of F. Crispo is also supported by the Program "Vanvitelli per la Ricerca: VALERE 2019" financed by the University of Campania "L. Vanvitelli". The research activity of C.R. Grisanti is performed under the auspices of GNAMPA-INdAM and partially supported by the Research Project of the University of Pisa "Energia e regolarità: tecniche innovative per problemi classici di equazioni alle derivate parziali". 
iii. for all $t, s \in(0, T)$, $v$ satisfies the equation:

$$
\begin{gathered}
\int_{s}^{t}\left[\left(v, \varphi_{\tau}\right)-(\nabla v, \nabla \varphi)+(v \cdot \nabla \varphi, v)\right] d \tau+(v(s), \varphi(s))=(v(t), \varphi(t)), \\
\text { for all } \varphi \in C_{0}^{1}([0, T) \times \Omega), \text { with } \nabla \cdot \varphi=0 .
\end{gathered}
$$

It is well known that any Leray's weak solution enjoys the energy inequality in strong form $[5,3,7]$ :

$$
\|v(t)\|_{2}^{2}+\int_{s}^{t}\|\nabla v(\tau)\|_{2}^{2} d \tau \leq\|v(s)\|_{2}^{2}, \text { for all } t>s, \text { a.e. in } s>0 \text { and for } s=0 .
$$

In the recent paper [2] the energy inequality (2) becomes a particular case of a suitable energy relation. In [2], assuming only $v_{0} \in J^{2}(\Omega)$, the existence of a suitable approximating sequence $\left\{v^{m}\right\}$ that, for all $p \in[1,2)$ and $T>0$, enjoys the strong convergence in $L^{p}\left(0, T ; J^{1,2}(\Omega)\right) \cap L^{2}\left(0, T ; J^{2}(\Omega)\right)$ to the weak solution $v$ of (1) is obtained. This is the first result of strong convergence of the gradient. Its interest is connected with the possibility of deducing an energy equality. This result surely holds if the convergence is strong in $L^{2}\left(0, T ; J^{1,2}(\Omega)\right)$. Of course this is not our case. The idea is to handle the strong convergence in $L^{p}\left(0, T ; J^{1,2}(\Omega)\right), p \in[1,2)$, suitably considering also the energy relation that holds for the approximation $\left\{v^{m}\right\}$. Unfortunately this it is not enough for the energy equality. Actually, by our arguments for the field $v$ only

$$
\|v(t)\|_{2}^{2}+2 \int_{s}^{t}\|\nabla v(\tau)\|_{2}^{2} d \tau-\|v(s)\|_{2}^{2}=-H(t, s), \text { a.e. in } t>s>0 \text { and for } s=0,
$$

and

$$
2 \int_{s}^{t}\|\nabla v(\tau)\|_{2}^{2} d \tau=F(t, s)\left(\|v(s)\|_{2}^{2}-\|v(t)\|_{2}^{2}\right), \text { a.e. in } t>s>0 \text { and for } s=0
$$

are fulfilled. The functions $H(t, s)$ and $F(t, s) \in(0,1]$ have the expressions:

$$
\begin{aligned}
& H(t, s):=\lim _{\gamma \rightarrow 0} \lim _{m \rightarrow \infty} \gamma \int_{s}^{t} \frac{\left\|v^{m}(\tau)\right\|_{2}^{2}}{\left(K+\left\|\nabla v^{m}(\tau)\right\|_{2}^{2}\right)^{\gamma}+1} \frac{d}{d \tau}\left\|\nabla v^{m}(\tau)\right\|_{2}^{2} d \tau, \text { for } s>0, \\
& H(t, 0):=\lim _{s \rightarrow 0} H(t, s)
\end{aligned}
$$

for any arbitrary constant $K>0$, as well as $v$ satisfies relation (4) with

$$
F(t, s):=\lim _{\gamma \rightarrow 0} \lim _{m \rightarrow \infty} \frac{1}{\left(K_{1}+\left\|\nabla v^{m}\left(t_{\gamma, m}\right)\right\|_{2}\right)} \gamma,
$$

for any arbitrary constant $K_{1} \geq 0$. Finally, the following property of inclusion holds: $\mathcal{G}_{1}:=$ $\{t, s$ such that (3) is true $\} \subseteq \mathcal{G}_{2}:=\{t, s$ such that (4) is true $\}$. Actually in paper [2] these sets of instants are the ones for which the strong convergence of the sequence $\left\{v^{m}\right\}$ holds in $J^{1,2}(\Omega)$ and in $J^{2}(\Omega)$, respectively. In the proof of formulas (7) and (11) we also use the set $\mathcal{G}_{1}$ as set of instants for which the quoted strong convergence holds. 
In this note we give further partial contributions for an energy equality that are of some interest. Firstly we provide a generalization of properties (3) and (4) respectively. Actually we are able to prove

$$
G(t, s)=\|v(s)\|_{2}^{2}-\|v(t)\|_{2}^{2}-2 \int_{s}^{t}\|\nabla v(\tau)\|_{2}^{2} d \tau, \text { a.e. in } t \geq s>0 \text { and for } s=0,
$$

and

$$
2 \int_{s}^{t}\|\nabla v(\tau)\|_{2}^{2} d \tau=P(t, s)\left(\|v(s)\|_{2}^{2}-\|v(t)\|_{2}^{2}\right), \text { a.e. in } t \geq s>0 \text { and for } s=0 .
$$

The functions $G(t, s)$ and $P(t, s)$ have the expressions:

$$
\begin{aligned}
& G(t, s):=-\lim _{\beta \rightarrow 0} \lim _{m \rightarrow \infty} \beta \int_{s}^{t}\left\|v^{m}(\tau)\right\|_{2}^{2} g^{\beta-1}\left(\left\|\nabla v^{m}(\tau)\right\|_{2}^{2}\right) g^{\prime}\left(\left\|\nabla v^{m}(\tau)\right\|_{2}^{2}\right) \frac{d}{d \tau}\left\|\nabla v^{m}(\tau)\right\|_{2}^{2} d \tau, \text { for } s>0, \\
& G(t, 0):=\lim _{s \rightarrow 0} G(t, s),
\end{aligned}
$$

for any arbitrary function $g(\rho)>0$ which is Liptchiz continuous on $[0, \infty)$ and such that $\lim _{\rho \rightarrow \infty} g(\rho)=0$, as well as $v$ satisfies relation (8) with alternative expressions for $P(t, s)$

$$
\lim _{R \rightarrow \infty} p_{R}^{\alpha}(h(\alpha, R)) \in(0,1], \lim _{\alpha \rightarrow 0^{+}} \lim _{R \rightarrow \infty} p_{R}^{\alpha}(h(\alpha, R)) \in(0,1], \lim _{\alpha \rightarrow \infty} \lim _{R \rightarrow \infty} p_{R}^{\alpha}(h(\alpha, R)) \in(0,1],
$$

where the exponent $\alpha$ is real, $p_{R}:[0, \infty) \rightarrow[0,1]$ is a positive continuous cut off function with $p_{R}(\rho)=1$ for $\rho \in[0, R], p_{R}(\rho)=0$ for $\rho \geq 2 R$, and $p_{R}(\rho) \in(0,1)$ for $\rho \in(R, 2 R)$, and finally $h(\alpha, R) \in[0,2 R)$. Of course by proving that $G(t, s) \leq 0$ or $P(t, s)=1$ one deduces the energy equality. No contraindication is known for these claims. Moreover we give the following

$$
E(t, s)=\|v(s)\|_{2}^{2}-\|v(t)\|_{2}^{2}-2 \int_{s}^{t}\|\nabla v(\tau)\|_{2}^{2} d \tau, \text { a.e. in } t \geq s>0 \text { and for } s=0,
$$

with

$$
\begin{aligned}
& E(t, s):=\lim _{R \rightarrow \infty} E(t, s, R) \begin{aligned}
E(t, s, R):=\lim _{m \rightarrow \infty}[ & {\left[\sum_{h=1}^{p_{22}(m, R)} 2\left[\left\|v^{m}\left(t_{h}^{22}\right)\right\|_{2}^{2}-\left\|v^{m}\left(s_{h}^{22}\right)\right\|_{2}^{2}\right]+\sum_{h=1}^{p_{21}(m, R)}\left[\left\|v^{m}\left(t_{h}^{21}\right)\right\|_{2}^{2}-2\left\|v^{m}\left(s_{h}^{21}\right)\right\|_{2}^{2}\right]\right.} \\
& \left.+\sum_{h=1}^{p_{11}(m, R)}\left[\left\|v^{m}\left(t_{h}^{11}\right)\right\|_{2}^{2}-\left\|v^{m}\left(s_{h}^{11}\right)\right\|_{2}^{2}\right]+\sum_{h=1}^{p_{12}(m, R)}\left[2\left\|v^{m}\left(t_{h}^{12}\right)\right\|_{2}^{2}-\left\|v^{m}\left(s_{h}^{12}\right)\right\|_{2}^{2}\right]\right]
\end{aligned} \\
& E(t, 0):=\lim _{s \rightarrow 0} E(t, s),
\end{aligned}
$$

where, for all $R>0, t_{h}^{i j}>s_{h}^{i j}$ and

$$
\sum_{h=1}^{p_{22}(m, R)}\left(t_{h}^{22}-s_{h}^{22}\right)+\sum_{h=1}^{p_{12}(m, R)}\left(t_{h}^{12}-s_{h}^{12}\right)+\sum_{h=1}^{p_{11}(m, R)}\left(t_{h}^{11}-s_{h}^{11}\right)+\sum_{h=1}^{p_{21}(m, R)}\left(t_{h}^{21}-s_{h}^{21}\right)<\frac{\left\|v_{0}\right\|_{2}^{2}}{2 R} .
$$

Since

$$
\left\|v^{m}\left(t_{h}^{i j}\right)\right\|_{2}-\left\|v^{m}\left(s_{h}^{i j}\right)\right\|_{2}<0, \text { for all } i, j, h
$$


we get that on the right-hand side of (12) only the last sum can be positive, the other sums surely are negative. In addition, by construction the equality

$$
p_{21}(m, R)=p_{12}(m, R)
$$

holds. Hence we get

$$
E(t, s, R)=\lim _{m \rightarrow \infty}\left[B(m, R)+\left\|v^{m}\left(t_{1}^{12}\right)\right\|_{2}^{2}-\left\|v^{m}\left(s_{p_{21}}^{21}\right)\right\|_{2}^{2}\right] \text {, with } B(m, R)<0 \text {, for all } R>0 .
$$

This is of some interest. Actually if $E(t, s) \leq 0$, then we trivially have that the energy equality holds.

In the linear case or for 2D-Navier-Stokes equations we get $G(t, s)=0, P(t, s)=1$ and $E(t, s)=0$. The first two claims are already proved in [2]. In the case of $E(t, s)$, for $R$ sufficiently large, we are able to prove that $p_{i j}(m, R)=0$ for all $i, j=1,2$.

All the above considerations are condensed in the following statement which is the chief result of the note:

Theorem 1. For all $v_{0} \in J^{2}(\Omega)$ there exists a weak solution $v$ to problem (1) which is a weakly continuous function of $t \geq 0$. The field $v$ is the weak ${ }^{*}$ limit in $L^{\infty}\left(0, T ; J^{2}(\Omega)\right)$ and weak limit in $L^{2}\left(0, T ; J^{1,2}(\Omega)\right)$ of a sequence $\left\{\left(v^{m}, \pi_{v^{m}}\right)\right\}$ of solutions to $(24)$. The sequence $\left\{v^{m}\right\}$ strongly converges to $v$ in $L^{2}\left(0, T ; L^{2}(\Omega)\right) \cap L^{p}\left(0, T ; W^{1,2}(\Omega)\right)$ for all $p \in[1,2)$. Moreover the limit $v$ satisfies the energy relations (7)-(11) with functions $G(t, s), P(t, s)$ and $E(t, s)$ given by (9)-(12) respectively. In particular a.e. in $s>0$ and in $s=0, v$ is continuous on the right in the $L^{2}$-norm. Finally, in the case of equality (11) estimates (13)-(15) hold.

REMARK 1. By virtue of (7)-(11) the energy inequality (2) is a particular case again. We stress that if the energy equality does not hold, then the gap with the equality is governed by the terms $G(t, s), P(t, s)$ and $E(t, s)$. Since for the definition of $G$ we have arbitrary auxiliary Lipschitz continuous functions $g(\rho)$ and for the definition of $P(t, s)$ arbitrary exponents $\alpha$ and its limits to 0 or $\infty$ respectively, we have a wide set of expressions related to the same value of the gap. In the case of the term $E(t, s)$, by means of a possible series, the gap is expressed by means of the $L^{2}$-norm of the approximations.

We remark that in the case of a bounded domain $\Omega$ the results also hold assuming the Galerkin approximation as sequence, as proposed in [8] and in [4].

Finally, if we consider the validity of the energy equality for the weak limit, for example by assuming, for all $\varepsilon>0, v \in L^{4}\left(\varepsilon, T ; L^{4}(\Omega)\right)$, as made in [6], which is compatible with the assumption $v_{0} \in J^{2}(\Omega)$, then we get $G(t, s)=E(t, s)=0$ and $P(t, s)=1$. It is of some interest to understand if in turn these new relations can imply an information on the partial regularity or on the singularity of the solution $v$, limit of the sequence $\left\{v^{m}\right\}$ quoted in Theorem 1.

\section{Notations and some preliminary result.}

The symbol $\mathscr{C}_{0}(\Omega)$ indicates the set of function $C_{0}^{\infty}(\Omega)$ and divergence free. In this paper we denote by $J^{2}(\Omega)$ and $J^{1,2}(\Omega)$ the completion spaces of $\mathscr{C}_{0}(\Omega)$ with respect to the metric of $L^{2}(\Omega)$ and $W^{1,2}(\Omega)$ respectively. By $L^{p}(0, T ; X)$ we mean the space of functions from $(0, T)$ into $X$ such that $\int^{T}$

$\int_{0}^{T}\|u(\tau)\|_{X}^{p} d \tau<\infty$. The following lemma is not known to the authors, hence the proof is proposed. 
Lemma 1. Let $\left\{h^{m}(t)\right\} \subset L^{1}((0, T))$ be a bounded sequence. Assume that $h^{m}(t) \rightarrow h(t)$ a.e. in $t \in(0, T)$ and $h \in L^{1}((0, T))$. Let $p(s) \in C([0, \infty))$ with $p(s)>0$ and $\lim _{s \rightarrow \infty} p(s)=0$. Then, there exists a function $h_{0}(t) \equiv h(t)$ a.e. in $t \in(0, T)$, such that, for all $\alpha>0$, we get

$$
\lim _{m} \int_{0}^{T}\left|h^{m}(t) p^{\alpha}\left(\left|h^{m}(t)\right|\right)-h(t) p^{\alpha}\left(\left|h_{0}(t)\right|\right)\right| d t=0 .
$$

Moreover, the following limit property also holds:

$$
\lim _{\alpha \rightarrow 0} \lim _{m} \int_{0}^{T} h^{m}(t) p^{\alpha}\left(\left|h^{m}(t)\right|\right) d t=\int_{0}^{T} h(t) d t .
$$

Proof. Since $h \in L^{1}((0, T))$, the estimate $|h(t)|<\infty$ holds for all $t \in(0, T)-A$ with meas $(A)=0$. We set $h_{0}(t)=h(t)$ for $t \in(0, T)-A$ and $h_{0}(t)=0$ for $t \in A$. We write the left-hand side of (16) as follows:

$$
\begin{aligned}
\int_{0}^{T} \mid h^{m}(t) & p^{\alpha}\left(\left|h^{m}(t)\right|\right)-h(t) p^{\alpha}\left(\left|h_{0}(t)\right|\right) \mid d t \\
& =\int_{0}^{T}\left|\left[h^{m}(t)-h(t)\right] p^{\alpha}\left(\left|h^{m}(t)\right|\right)+h(t)\left[p^{\alpha}\left(\left|h^{m}(t)\right|\right)-p^{\alpha}\left(\left|h_{0}(t)\right|\right)\right]\right| d t \\
\leq & \int_{0}^{T}\left|h^{m}(t)-h(t)\right| p^{\alpha}\left(\left|h^{m}(t)\right|\right) d t+\int_{0}^{T}|h(t)|\left|p^{\alpha}\left(\left|h^{m}(t)\right|\right)-p^{\alpha}\left(\left|h_{0}(t)\right|\right)\right| d t .
\end{aligned}
$$

Since $\left|p^{\alpha}\left(\left|h^{m}(t)\right|\right)-p^{\alpha}\left(\left|h_{0}(t)\right|\right)\right| \leq 2\|p\|_{\infty}^{\alpha}$, for all $t \in(0, T)$, and $h^{m}(t) \rightarrow h(t)$ a.e. in $t \in(0, T)$, applying the Lebesgue dominated convergence theorem, we arrive at

$$
\lim _{m} \int_{0}^{T}|h(t)|\left|p^{\alpha}\left(\left|h^{m}(t)\right|\right)-p^{\alpha}\left(\left|h_{0}(t)\right|\right)\right| d t=0 .
$$

Concerning the first integral on the right-hand side of (18), we arrange the following artifice:

$$
\begin{aligned}
\int_{0}^{T}\left|h^{m}(t)-h(t)\right| p^{\alpha}\left(\left|h^{m}(t)\right|\right) d t & =\int_{I_{M}^{m}}\left|h^{m}(t)-h(t)\right| p^{\alpha}\left(\left|h^{m}(t)\right|\right) d t+\int_{(0, T)-I_{M}^{m}}\left|h^{m}(t)-h(t)\right| p^{\alpha}\left(\left|h^{m}(t)\right|\right) d t \\
& =: J_{1}^{m}+J_{2}^{m},
\end{aligned}
$$

where, for some fixed $M>0, I_{M}^{m}:=\left\{t:\left|h^{m}(t)\right| \leq M\right\}$. By the definition of $I_{M}^{m}$, we easily obtain

$$
J_{2}^{m} \leq p_{M}^{\alpha} \int_{0}^{T}\left(\left|h^{m}(t)\right|+|h(t)|\right) d t \leq c p_{M}^{\alpha}, \text { for all } m \in \mathbb{N},
$$

where $c$ is independent of $M$ and $p_{M}:=\sup _{(M, \infty)} p(s)$. Concerning $J_{1}^{m}$, we introduce the characteristic function of the set $I_{M}^{m}$, so that

$$
H^{m}(t):=\left|h^{m}(t)-h(t)\right| p^{\alpha}\left(\left|h^{m}(t)\right|\right) \chi_{I_{M}^{m}}(t) \rightarrow 0 \text { a.e. in } t \in(0, T)
$$$$
\text { and, a.e. in } t \in(0, T), H^{m}(t) \leq(M+|h(t)|)\|p\|_{\infty}^{\alpha} \text { for all } m \in \mathbb{N} \text {. }
$$ 
So that applying the Lebesgue dominated convergence theorem, we deduce that $\lim _{m} J_{1}^{m}=0$. The above limit property of $J_{1}^{m}$ and estimate on $J_{2}^{m}$, via (18)-(19), furnish

$$
\lim _{m} \int_{0}^{T}\left|h^{m}(t) p^{\alpha}\left(\left|h^{m}(t)\right|\right)-h(t) p^{\alpha}\left(\left|h_{0}(t)\right|\right)\right| d t \leq c p_{M}^{\alpha} \text {, for all } M>0 .
$$

Since $p \rightarrow 0$ for $s \rightarrow \infty$, letting $M \rightarrow \infty$ we prove (16). By applying the Lebesgue dominated convergence theorem we immediately get (17).

Corollary 1. Let $\left\{h^{m}(t)\right\} \subset L^{1}((0, T))$ be a sequence with $A:=\sup \left\|h^{m}\right\|_{1}<\infty$. Assume that $h^{m}(t) \rightarrow h(t)$ a.e. in $t \in(0, T)$ and $h \in L^{1}((0, T))$. Let $p_{R}(s) \in C([0, \infty))$ with $p_{R}(s)=1$ for $s \leq R$, $p_{R}(s)=0$ for $s \geq 2 R$ and $p_{R}(s) \in(0,1)$ for $s \in(R, 2 R)$. Then, there exists a function $h_{0}(t) \equiv h(t)$ a.e. in $t \in(0, T)$, such that, for all $R>(2 T)^{-1} A$, we get

$$
\lim _{m} \int_{0}^{T}\left|h^{m}(t) p_{R}\left(\left|h^{m}(t)\right|\right)-h(t) p_{R}\left(\left|h_{0}(t)\right|\right)\right| d t=0 .
$$

Moreover, the following limit also holds:

$$
\lim _{R \rightarrow \infty} \lim _{m} \int_{0}^{T} h^{m}(t) p_{R}\left(\left|h^{m}(t)\right|\right) d t=\int_{0}^{T} h(t) d t
$$

Proof. The first claim of the corollary is a particular case of the previous lemma. Hence we get

$$
\begin{aligned}
\int_{0}^{T} \mid h^{m}(t) & p_{R}\left(\left|h^{m}(t)\right|\right)-h(t) p_{R}\left(\left|h_{0}(t)\right|\right) \mid d t \\
\quad & \int_{0}^{T}\left|\left[h^{m}(t)-h(t)\right] p_{R}\left(\left|h^{m}(t)\right|\right)+h(t)\left[p_{R}\left(\left|h^{m}(t)\right|\right)-p_{R}\left(\left|h_{0}(t)\right|\right)\right]\right| d t \\
\leq & \int_{0}^{T}\left|h^{m}(t)-h(t)\right| p_{R}\left(\left|h^{m}(t)\right|\right) d t+\int_{0}^{T}|h(t)|\left|p_{R}\left(\left|h^{m}(t)\right|\right)-p_{R}\left(\left|h_{0}(t)\right|\right)\right| d t,
\end{aligned}
$$

and

$$
\lim _{m} \int_{0}^{T}|h(t)|\left|p_{R}\left(\left|h^{m}(t)\right|\right)-p_{R}\left(\left|h_{0}(t)\right|\right)\right| d t=0 .
$$

Concerning the first integral on the right-hand side of (22), we get

$$
\int_{0}^{T}\left|h^{m}(t)-h(t)\right| p_{R}\left(\left|h^{m}(t)\right|\right) d t=\int_{T_{R}}\left|h^{m}(t)-h(t)\right| p_{R}\left(\left|h^{m}(t)\right|\right) d t=: J_{R}^{m},
$$


where we set $T_{R}^{m}:=\left\{t: 0 \leq h^{m}(t)<2 R\right\}^{1}$. Since

$$
\begin{gathered}
H^{m}(t):=\left|h^{m}(t)-h(t)\right| p_{R}\left(\left|h^{m}(t)\right|\right) \rightarrow 0 \text { a.e. in } t \in T_{R}^{m}, \\
H^{m}(t) \leq 2 R, \text { for all } m \in \mathbb{N} \text { and } t \in T_{R}^{m},
\end{gathered}
$$

applying the Lebesgue dominated convergence theorem, we deduce that, for all $R>0$, letting $m \rightarrow \infty$, $J_{R}^{m} \rightarrow 0$. The above limit property via (22)-(23) furnishes

$$
\lim _{m} \int_{0}^{T}\left|h^{m}(t) p_{R}\left(\left|h^{m}(t)\right|\right)-h(t) p_{R}\left(\left|h_{0}(t)\right|\right)\right| d t=0,
$$

hence

$$
\lim _{m} \int_{0}^{T} h^{m}(t) p_{R}\left(\left|h^{m}(t)\right|\right)=\int_{0}^{T} h(t) p_{R}\left(\left|h_{0}(t)\right|\right) d t d t .
$$

The first limit proves (20). By applying Lebesgue's theorem to the second identity, letting $R \rightarrow \infty$, we immediately get (21).

We consider a mollified Navier-Stokes initial boundary value problem:

$$
\begin{aligned}
& v_{t}^{m}+J_{m}\left[v^{m}\right] \cdot \nabla v^{m}+\nabla \pi_{v^{m}}=\Delta v^{m}, \nabla \cdot v^{m}=0, \text { in }(0, T) \times \Omega, \\
& v^{m}=0 \text { on }(0, T) \times \partial \Omega, v^{m}(0, x)=v_{0}^{m}(x) \text { on }\{0\} \times \Omega,
\end{aligned}
$$

where $J_{m}[\cdot]$ is a mollifier and $\left\{v_{0}^{m}\right\} \subset J^{1,2}(\Omega)$ converges to $v_{0}$ in $J^{2}(\Omega)$. In [2] is proved

Lemma 2. For all $v_{0} \in J^{2}(\Omega)$ there exists a sequence of solutions $\left\{\left(v^{m}, \pi_{v^{m}}\right)\right\}$ such that, for all $m \in \mathbb{N}$ and $T>0, v^{m} \in C\left([0, T) ; J^{1,2}(\Omega)\right) \cap L^{2}\left(0, T ; W^{2,2}(\Omega)\right)$.

Lemma 3. The sequence $\left\{v^{m}\right\}$ of kinetic fields of Lemma 2 admits limit $v$ which is weak* limit in $L^{\infty}\left(0, T ; J^{2}(\Omega)\right)$, weak limit in $L^{2}\left(0, T ; J^{1,2}(\Omega)\right)$ and, for all $p \in[1,2)$, strong limit in $L^{p}\left(0, T ; J^{1,2}\right) \cap$ $L^{2}\left(0, T ; J^{2}(\Omega)\right)$. Moreover, $v$ is a weak solution to problem $(1)$ with $(v(t), \psi)$ continuous function of $t$, for all $\psi \in J^{2}(\Omega)$.

Proof. Apart from the strong convergence properties, the result is well known. The properties of strong convergence are proved in [2].

Lemma 4. Let $\left(\left\{v^{m}, \pi_{v_{m}}\right)\right\}$ be the sequence furnished by Lemma 2. Then, for all $t \geq 0$ and $m \in \mathbb{N}$, $\left\|v^{m}\right\|_{1,2} \neq 0$ holds.

Proof. See Lemma 10 in [2].

Lemma 5. Let $v$ be a weak solution. Assume that $\|v(s)\|_{2} \neq 0$ and $v$ is right continuous in the $L^{2}$-norm. Then there exists $\delta>0$ such that $\|v(t)\|_{2} \neq 0$ for all $t \in[s, s+\delta)$.

Proof. The proof is given in [2]. However since it is very short, for the sake of the completeness we reproduce it. Assume that for all $\delta>0$ we have a $t \in(s, s+\delta)$ such that $\|v(t)\|_{2}=0$. Then we can select a sequence $\left\{t_{p}\right\}$ converging to $s$ such that $\left\|v\left(t_{p}\right)\right\|=0$. Then, by virtue of the right- $L^{2}$-continuity in $s$, we get $\lim _{t_{p} \rightarrow s^{+}}\left\|v\left(t_{p}\right)-v(s)\right\|_{2}=0$, which is a contradiction with $\|v(s)\|_{2} \neq 0$.

\footnotetext{
${ }^{1}$ We stress that, for all $m \in \mathbb{N}$, meas $\left(T_{R}^{m}\right) \neq 0$. Actually, if for $2 T R>A$ we guess that $m e a s\left(T_{R}^{m}\right)=0$, then

$$
A<\int_{0}^{T} 2 R \leq \int_{0}^{T} h^{m}(t) d t \leq A .
$$
}




\section{Proof of Theorem 1}

Taking into account the results of Lemma 2 and Lemma3, our proof is reduced to prove the energy inequalities (7)-(8). In the sequel by $\mathcal{G}_{1}$ we mean the set

$$
\left\{t \geq 0:\left\|v^{m}(t)-v(t)\right\|_{1,2} \rightarrow 0 \text { as } m \rightarrow \infty\right\} .
$$

Thanks to Lemma 3 we have meas $\left(\mathbb{R}^{+}-\mathcal{G}_{1}\right)=0$. In the sequel by $\mathcal{G}_{2}$ we mean the set

$$
\left\{t \geq 0:\left\|v^{m}(t)-v(t)\right\|_{2} \rightarrow 0 \text { as } m \rightarrow \infty\right\} .
$$

We have meas $\left(\mathbb{R}^{+}-\mathcal{G}_{2}\right)=0$, and a priori $\mathcal{G}_{1} \subseteq \mathcal{G}_{2}$ holds.

Energy equality (7).

The starting point is the energy differential equation of $\left(v^{m}, \pi_{v^{m}}\right)$ related to the solution of the problem (24), that is

$$
\frac{d}{d t}\left\|v^{m}(t)\right\|_{2}^{2}+2\left\|\nabla v^{m}(t)\right\|_{2}^{2}=0, t \geq 0 .
$$

We multiply equation (25) by $g^{\beta}\left(\left\|\nabla v^{m}(t)\right\|_{2}^{2}\right)$. Here $g(\rho)>0$ is a Lipschitz continuous function of $\rho \geq 0$ such that $\lim _{\rho \rightarrow \infty} g(\rho)=0$ and $\beta>0$ is an exponent. We consider $s, t \in \mathcal{G}_{1}$ with $0<s<t$. Integrating by parts on the interval $(s, t)$, we get

$$
\begin{aligned}
& -\beta \int_{s}^{t}\left\|v^{m}(\tau)\right\|_{2}^{2} g^{\beta-1}\left(\left\|\nabla v^{m}(\tau)\right\|_{2}^{2}\right) g^{\prime}\left(\left\|\nabla v^{m}(\tau)\right\|_{2}^{2}\right) \frac{d}{d t}\left\|\nabla v^{m}(\tau)\right\|_{2}^{2} d \tau \\
& \quad=\left\|v^{m}(s)\right\|_{2}^{2} g^{\beta}\left(\left\|\nabla v^{m}(s)\right\|_{2}^{2}\right)-\left\|v^{m}(t)\right\|_{2}^{2} g^{\beta}\left(\left\|\nabla v^{m}(t)\right\|_{2}^{2}\right)-2 \int_{s}^{t} g^{\beta}\left(\left\|\nabla v^{m}(\tau)\right\|_{2}^{2}\right)\left\|\nabla v^{m}(\tau)\right\|_{2}^{2} d \tau .
\end{aligned}
$$

Recalling the definition of $\mathcal{G}_{1}$, by virtue of Lemma 1 , letting $m \rightarrow \infty$ and subsequently letting $\beta \rightarrow 0$, we arrive at (7) with $G(t, s)$ given by (9).

Energy equality (8).

We consider $s, t \in \mathcal{G}_{2}$ with $0 \leq s<t$. We introduce a cutoff function $p_{R}(\rho)$ which is Lipschitz continuous and such that $p_{R}(\rho)=1$ for $\rho \in[0, R], p_{R}(\rho)=0$ for $\rho \geq 2 R$ and $p_{R}(\rho) \in(0,1)$ for $\rho \in(R, 2 R)$. We set

$$
A=\int_{0}^{t}\|\nabla v(\tau)\|_{2}^{2} d \tau, \text { and } R_{0}>(2 t)^{-1} A \text { and such that meas }\left(\left\{\tau \in(0, t):\|\nabla v(\tau)\|_{2}^{2}<2 R_{0}\right\}\right) \neq 0 .
$$

The claim (27) is consistent by virtue of Lemma 5 . For $\alpha>0$, we get

$$
p_{R}^{\alpha}\left(\left\|\nabla v^{m}(t)\right\|_{2}^{2}\right) \frac{1}{2} \frac{d}{d t}\left\|v^{m}(t)\right\|_{2}^{2}+p_{R}^{\alpha}\left(\left\|\nabla v^{m}(t)\right\|_{2}^{2}\right)\left\|\nabla v^{m}(t)\right\|_{2}^{2}=0 .
$$

Integrating on $(0, t)$, considering $R>R_{0}$ and applying the mean value theorem, for some $t_{\alpha, R}^{m} \in(0, t)$, we get

$$
p_{R}^{\alpha}\left(\left\|\nabla v^{m}\left(t_{\alpha, R}^{m}\right)\right\|_{2}^{2}\right) \frac{1}{2}\left[\left\|v^{m}(t)\right\|_{2}^{2}-\left\|v^{m}(0)\right\|_{2}^{2}\right]=-\int_{0}^{t} p_{R}^{\alpha}\left(\left\|\nabla v^{m}(\tau)\right\|_{2}^{2}\right)\left\|\nabla v^{m}(\tau)\right\|_{2}^{2} d \tau .
$$


By virtue of Lemma 4 , for all $m \in \mathbb{N}$ the right-hand side is different from to zero, we deduce that $\left\|\nabla v^{m}\left(t_{\alpha, R}^{m}\right)\right\|_{2}^{2} \in(0,2 R)$ for all $m \in \mathbb{N}$. Hence there exists an extract which admits a limit $h(\alpha, R) \in$ $[0,2 R]$. We label again by $m$ the extract sequence. Letting $m \rightarrow \infty$, firstly we obtain

$$
\lim _{m} p_{R}^{\alpha}\left(\left\|\nabla v^{m}\left(t_{\alpha, R}^{m}\right)\right\|_{2}^{2}\right)=\frac{2 \int_{0}^{t} p_{R}^{\alpha}\left(\|\nabla v(\tau)\|_{2}^{2}\right)\|\nabla v(\tau)\|_{2}^{2} d \tau}{\|v(0)\|_{2}^{2}-\|v(t)\|_{2}^{2}} \neq 0
$$

as a consequence of (27). Then, since, for all $\alpha>0$ and $R>R_{0}, p_{R}^{\alpha}$ is continuous, we get $\lim _{m} p_{R}^{\alpha}\left(\left\|\nabla v^{m}\left(t_{\alpha, R}^{m}\right)\right\|_{2}^{2}\right)=p_{R}^{\alpha}(h(\alpha, R))$. So that letting $R \rightarrow \infty$, we get

$$
\lim _{R} p_{R}^{\alpha}(h(\alpha, R))=\frac{2 \int_{0}^{t}\|\nabla v(\tau)\|_{2}^{2} d \tau}{\|v(0)\|_{2}^{2}-\|v(t)\|_{2}^{2}} \neq 0,
$$

which proves (8) with $P(t, s)$ given by $(10)_{1}$. The alternative expressions of $P(t, s)$ are an immediate consequence of the fact that the right-hand side is independent of $\alpha$.

Energy equality (11).

We consider $s, t \in \mathcal{G}_{1}$ with $0<s<t$. We consider equation (28) with $p_{R}(\rho):=\frac{2 R-\rho}{R}$, where $R>\max \left\{\|\nabla v(s)\|_{2}^{2},\|\nabla v(t)\|_{2}^{2}\right\}$. Since $s, t \in \mathcal{G}_{1}$ we have strong convergence in $J^{1,2}(\Omega)$ of $\left\{v^{m}(s, x)\right\}$ to $v(s, x)$ and of $\left\{v^{m}(t, x)\right\}$ to $v(t, x)$, respectively. Hence, without losing the generality, we also claim that $R>\max \left\{\left\|\nabla v^{m}(s)\right\|_{2}^{2},\left\|\nabla v^{m}(t)\right\|_{2}^{2}\right\}$ for all $m \in \mathbb{N}$. Integrating on $(s, t)$, after an integration by parts we get

$$
-\int_{s}^{t}\left\|v^{m}(\tau)\right\|_{2}^{2} \frac{d}{d \tau} p_{R}\left(\left\|\nabla v^{m}(\tau)\right\|_{2}^{2}\right) d \tau=\left\|v^{m}(s)\right\|_{2}^{2}-\left\|v^{m}(t)\right\|_{2}^{2}-2 \int_{s}^{t} p_{R}\left(\left\|\nabla v^{m}(\tau)\right\|_{2}^{2}\right)\left\|\nabla v^{m}(\tau)\right\|_{2}^{2} d \tau .
$$

We set

$$
I_{R}^{m}:=\left\{\tau \in(s, t): R<\left\|\nabla v^{m}(\tau)\right\|_{2}^{2}<2 R\right\} .
$$

The set $I_{R}^{m}$, if not empty, is at most a countable union of disjoint open intervals. Actually, let $J$ be the interior set of $(s, t)-I_{R}^{m}$. Since $\partial I_{R}^{m}$ is at most countable, we get

$$
\int_{s}^{t}\left\|v^{m}(\tau)\right\|_{2}^{2} \frac{d}{d \tau} p_{R}\left(\left\|\nabla v^{m}(\tau)\right\|_{2}^{2}\right) d \tau=\int_{I_{R}^{m}}\left\|v^{m}(\tau)\right\|_{2}^{2} \frac{d}{d \tau} p_{R}\left(\left\|\nabla v^{m}(\tau)\right\|_{2}^{2}\right) d \tau+\int_{J}\left\|v^{m}(\tau)\right\|_{2}^{2} \frac{d}{d \tau} p_{R}\left(\left\|\nabla v^{m}(\tau)\right\|_{2}^{2}\right) d \tau .
$$

Let us fix $\bar{\tau} \in J$ and suppose that $\left\|\nabla v^{m}(\bar{\tau})\right\|_{2}^{2} \leq R$. Since $J$ is open, it contains an interval centered at $\bar{\tau}$ where, by continuity, $\left\|\nabla v^{m}(\tau)\right\|_{2}^{2} \leq R$ holds. Hence we get $\frac{d}{d \tau} p_{R}\left(\left\|v^{m}(\tau)\right\|_{2}^{2}\right)=0$. The same argument works in the case of $\left\|\nabla v^{m}(\bar{\tau})\right\|_{2}^{2} \geq 2 R$. Thus, we finally get

$$
\int_{s}^{t}\left\|v^{m}(\tau)\right\|_{2}^{2} \frac{d}{d \tau} p_{R}\left(\left\|\nabla v^{m}(\tau)\right\|_{2}^{2}\right) d \tau=\int_{I_{R}^{m}}\left\|v^{m}(\tau)\right\|_{2}^{2} \frac{d}{d \tau} p_{R}\left(\left\|\nabla v^{m}(\tau)\right\|_{2}^{2}\right) d \tau,
$$

which coupled with (29) furnish

$$
\int_{I_{R}^{m}} \frac{\left\|v^{m}(\tau)\right\|_{2}^{2}}{R} \frac{d}{d \tau}\left\|\nabla v^{m}(\tau)\right\|_{2}^{2} d \tau=\left\|v^{m}(s)\right\|_{2}^{2}-\left\|v^{m}(t)\right\|_{2}^{2}-2 \int_{s}^{t} p_{R}\left(\left\|\nabla v^{m}(\tau)\right\|_{2}^{2}\right)\left\|\nabla v^{m}(\tau)\right\|_{2}^{2} d \tau .
$$


We denote by $p_{i j}(m, R), i, j=1,2$, a positive or null integer. If we have $i \neq j$, then $p_{i j}(m, R) \in \mathbb{N}$ (see item 3 . below). If we have $i=j$, then $p_{i i}(m, R)$ can be $\infty$. Actually, we distinguish the intervals of the union in the following way:

- $h \in\left\{1, \ldots, p_{11}(m, R)\right\}$, where a priori $p_{11}(m, R) \in \mathbb{N} \cup\{\infty\},\left(s_{h}^{11}, t_{h}^{11}\right)$ is the interval such that $\left\|\nabla v^{m}(\tau)\right\|_{2}^{2} \in(R, 2 R)$, for all $\tau \in\left(s_{h}^{11}, t_{h}^{11}\right)$, with $\left\|\nabla v^{m}\left(s_{h}^{11}\right)\right\|_{2}^{2}=\left\|\nabla v^{m}\left(t_{h}^{11}\right)\right\|_{2}^{2}=R$,

- $h \in\left\{1, \ldots, p_{12}(m, R)\right\},\left(s_{h}^{12}, t_{h}^{12}\right)$ is the interval such that $\left\|\nabla v^{m}(\tau)\right\|_{2}^{2} \in(R, 2 R)$, for all $\tau \in$ $\left(s_{h}^{12}, t_{h}^{12}\right)$, with $\left\|\nabla v^{m}\left(s_{h}^{12}\right)\right\|_{2}^{2}=R$ and $\left\|\nabla v^{m}\left(t_{h}^{12}\right)\right\|_{2}^{2}=2 R$,

- $h \in\left\{1, \ldots, p_{21}(m, R)\right\},\left(s_{h}^{21}, t_{h}^{21}\right)$ is the interval such that $\left\|\nabla v^{m}(\tau)\right\|_{2}^{2} \in(R, 2 R)$, for all $\tau \in$ $\left(s_{h}^{21}, t_{h}^{21}\right)$, with $\left\|\nabla v^{m}\left(s_{h}^{21}\right)\right\|_{2}^{2}=2 R$ and $\left\|\nabla v^{m}\left(s_{h}^{21}\right)\right\|_{2}^{2}=R$,

- $h \in\left\{1, \ldots, p_{22}(m, R)\right\}$, where a priori $p_{22}(m, R) \in \mathbb{N} \cup\{\infty\},\left(s_{h}^{22}, t_{h}^{22}\right)$ is the interval such that $\left\|\nabla v^{m}(\tau)\right\|_{2}^{2} \in(R, 2 R)$, for all $\tau \in\left(s_{h}^{22}, t_{h}^{22}\right)$, with $\left\|\nabla v^{m}\left(s_{h}^{22}\right)\right\|_{2}^{2}=\left\|\nabla v^{m}\left(t_{h}^{22}\right)\right\|_{2}^{2}=2 R$.

Since $\left\|\nabla v^{m}(s)\right\|_{2}^{2}<R$ and $\left\|\nabla v^{m}(t)\right\|_{2}^{2}<R$, we deduce that $s<\min s_{h}^{i j}$ and $t>\max t_{h}^{i j}$. Moreover

1. $p_{i j}(m, R)=0$, for all $i, j \in\{1,2\}$, for all $m \in \mathbb{N}$ if, and only if, $\left\|\nabla v^{m}(\tau)\right\|_{2}^{2}<R$ for all $m \in \mathbb{N}$.

2. $p_{12}(m, R)=p_{21}(m, R)=p_{22}(m, R)=0$ if, and only if, $\left\|\nabla v^{m}(\tau)\right\|_{2}^{2}<2 R$ for all $\tau \in(s, t)$.

3. $p_{12}(m, R) \neq 0$ if, and only if, $p_{21}(m, R) \neq 0$, and in both cases $p_{i j}(m, R) \in \mathbb{N}$. Actually, we have that $\left\{\left(s_{h}^{12}, t_{h}^{12}\right)\right\}$ is a sequence, the sequence collapses into a point $\bar{t}$ and as a consequence we get $R=\lim _{s_{h}^{12} \rightarrow \bar{t}} p_{R}\left(\left\|\nabla v^{m}\left(s_{h}^{12}\right)\right\|_{2}^{2}\right)=p_{R}\left(\left\|\nabla v^{m}(\bar{t})\right\|_{2}^{2}\right)=\lim _{t_{h}^{12} \rightarrow \bar{t}} p_{R}\left(\left\|\nabla v^{m}\left(t_{h}^{12}\right)\right\|_{2}^{2}\right)=2 R$ which is a contradiction. Analogous argument works in the case of " 21 ".

4. We have $p_{12}(m, R)=p_{21}(m, R)$. Actually, for fixed $h \leq p_{12}(m, R)$, let us consider the set $A:=\left\{k \in \mathbb{N}: t_{h}^{12} \leq s_{k}^{21}\right\}$. Since $\left\|\nabla v^{m}(t)\right\|_{2}^{2}<R$, by continuity, the set $A$ is not empty. Let be $\varphi(h):=\min (A)$. Then, by construction, $\left\|\nabla v^{m}(\tau)\right\|_{2}^{2}>R$ for all $\tau$ such that $\tau \in\left(t_{h}^{12}, t_{\varphi(h)}^{21}\right)$. It follows that

$$
s_{\varphi(h)}^{21}<t_{\varphi(h)}^{21}<s_{h+1}^{12}<t_{h+1}^{12}<s_{\varphi(h+1)}^{21} .
$$

Hence function $\varphi$ is injective. Conversely, using the fact that $\left\|\nabla v^{m}(s)\right\|_{2}^{2}<R$, we can conclude that $p_{12}(m, R)=p_{21}(m, R)$.

Since $2 \int_{s}^{t}\left\|\nabla v^{m}(\tau)\right\|_{2}^{2} d \tau \leq\left\|v_{0}\right\|_{2}^{2}$, then, for all $m \in \mathbb{N}$ and $R$, we get meas $\left(I_{R}^{m}\right)<\frac{\left\|v_{0}\right\|_{2}^{2}}{2 R}$ which proves (13). By the energy inequality the same property holds for the weak solution $v$, that is

$$
I_{R}:=\left\{\tau \in(s, t): R \leq\|\nabla v(\tau)\|_{2}^{2} \leq 2 R\right\}, \text { then meas }\left(I_{R}\right)<\frac{\left\|v_{0}\right\|_{2}^{2}}{2 R} .
$$

Taking into account the previous considerations, integrating by parts on the intervals defining $I_{R}^{m}$, we get

$$
E^{m}=-\frac{2}{R} \int_{I_{R}^{m}}\left\|\nabla v^{m}(\tau)\right\|_{2}^{4} d \tau+\left\|v^{m}(s)\right\|_{2}^{2}-\left\|v^{m}(t)\right\|_{2}^{2}-2 \int_{s}^{t} p_{R}\left(\left\|\nabla v^{m}(t)\right\|_{2}^{2}\right)\left\|\nabla v^{m}(\tau)\right\|_{2}^{2} d \tau
$$


where we considered that $\frac{d}{d t}\left\|v^{m}(t)\right\|_{2}^{2}=-2\left\|\nabla v^{m}(t)\right\|_{2}^{2}$, and we set

$$
\begin{aligned}
E^{m}:=\sum_{h=1}^{p_{22}(m, R)} 2\left[\left\|v^{m}\left(t_{h}^{22}\right)\right\|_{2}^{2}-\left\|v^{m}\left(s_{h}^{22}\right)\right\|_{2}^{2}\right]+\sum_{h=1}^{p_{21}(m, R)}\left[\left\|v^{m}\left(t_{h}^{21}\right)\right\|_{2}^{2}-2\left\|v^{m}\left(s_{h}^{21}\right)\right\|_{2}^{2}\right] \\
+\sum_{h=1}^{p_{11}(m, R)}\left[\left\|v^{m}\left(t_{h}^{11}\right)\right\|_{2}^{2}-\left\|v^{m}\left(s_{h}^{11}\right)\right\|_{2}^{2}\right]+\sum_{h=1}^{p_{12}(m, R)}\left[2\left\|v^{m}\left(t_{h}^{12}\right)\right\|_{2}^{2}-\left\|v^{m}\left(s_{h}^{12}\right)\right\|_{2}^{2}\right] .
\end{aligned}
$$

In the expression of $E^{m}$ we have taken into account that the value of $\left\|\nabla v^{m}(\tau)\right\|_{2}^{2}$ in the end points of the intervals is alternatively $R$ or $2 R$ and for all the integral term there is the moltiplicative factor $\frac{1}{R}$. We discuss the first integral on the right-hand side of (31). By the definition of $I_{R}^{m}$ we deduce that $\frac{\left\|\nabla v^{m}(t)\right\|_{2}^{2}}{R} \leq 2$ for all $\tau \in I_{R}^{m}$ and for all $m \in \mathbb{N}$. Hence, employing the energy relation for $v^{m}$ and the Lebesgue theorem, we deduce that for all $R$

$$
\lim _{m} \frac{1}{R} \int_{I_{R}^{m}}\left\|\nabla v^{m}(\tau)\right\|_{2}^{4} d \tau \leq 2 \lim _{m} \int_{I_{R}^{m}}\left\|\nabla v^{m}(\tau)\right\|_{2}^{2} d \tau \leq 2 \int_{I_{R}}\|\nabla v(\tau)\|_{2}^{2} d \tau .
$$

Since for all $T>0$ we have $\|\nabla v(\tau)\|_{2}^{2} \in L^{1}(0, T)$, then, via (30), we get

$$
\lim _{R} \frac{1}{R} \lim _{m} \int_{I_{R}^{m}}\left\|\nabla v^{m}(\tau)\right\|_{2}^{4} d \tau \leq 2 \lim _{R} \int_{I_{R}}\|\nabla v(t)\|_{2}^{2} d \tau=0 .
$$

Letting $m \rightarrow \infty$ and then letting $R \rightarrow \infty$ in formula (31), we arrive at (11) with $E(t, s)$ given by (12). Finally, we justify (15). First of all we stress that by virtue of the energy inequality $\left\|v^{m}(t)\right\|_{2}<\left\|v^{m}(s)\right\|_{2}$ true for all $t>s$ and $m \in \mathbb{N}$, we get that in (32) only the last sum can be positive. Concerning (15) it is enough to restrict ourselves to the finite sum with indexes $h \in$ $\left\{1, \ldots, p_{12}(m, R)\right\} \cup\left\{1, \ldots, p_{21}(m, R)\right\}$. Taking into account that $p_{12}(m, R)=p_{21}(m, R)$, for all $h \in\left\{1, \ldots, p_{12}(m, R)\right\}$ we have

$$
\begin{gathered}
\left\|v^{m}\left(t_{h}^{12}\right)\right\|_{2}^{2}-\left\|v^{m}\left(s_{h}^{12}\right)\right\|_{2}^{2}<0, \\
\left\|v^{m}\left(t_{h}^{21}\right)\right\|_{2}^{2}-\left\|v^{m}\left(s_{h}^{21}\right)\right\|_{2}^{2}<0 .
\end{gathered}
$$

Finally, if $h \in\left\{2, \ldots, p_{12}(m, R)\right\}$, then the following holds:

$$
\left\|v^{m}\left(t_{h}^{12}\right)\right\|_{2}^{2}-\left\|v^{m}\left(s_{h-1}^{21}\right)\right\|_{2}^{2}<0 .
$$

Hence we obtain

$$
\begin{aligned}
\sum_{h=1}^{p_{21}(m, R)}\left[\left\|v^{m}\left(t_{h}^{21}\right)\right\|_{2}^{2}-2\left\|v^{m}\left(s_{h}^{21}\right)\right\|_{2}^{2}\right]+\sum_{h=1}^{p_{12}(m, R)}\left[2\left\|v^{m}\left(t_{h}^{12}\right)\right\|_{2}^{2}-\left\|v^{m}\left(s_{h}^{12}\right)\right\|_{2}^{2}\right] \\
=B(m, R)+\left\|v^{m}\left(t_{1}^{12}\right)\right\|_{2}^{2}-\left\|v^{m}\left(s_{p_{21}}^{21}\right)\right\|_{2}^{2},
\end{aligned}
$$

with $B(m, R)<0$ and $t_{1}^{12}<s_{p_{21}}^{21}$.

\section{References}

[1] L. Caffarelli, R. Kohn, and L. Nirenberg, Partial regularity of suitable weak solutions of the Navier-Stokes equations, Comm. Pure Appl. Math. 35 (1982), no. 6, 771-831. 
[2] F. Crispo, C.R. Grisanti and P. Maremonti, Some new properties of a suitable weak solution to the NavierStokes equations ArXiv:1904.07641, to appear in Waves in Flows: The 2018 Prague- Sum Workshop Lectures, series: Lecture Notes in Mathematical Fluids Mechanics, editors: G.P.Galdi, T. Bodnar, S. Necasova, Birkhauser.

[3] G.P. Galdi and P. Maremonti, Monotonic decreasing and asymptotic behavior of the kinetic energy for weak solutions of the Navier-Stokes equations in exterior domains, Arch. Rational Mech. Anal. 94 (1986), no. 3, 253-266.

[4] J.G. Heywood, The Navier-Stokes equations: on the existence, regularity and decay of solutions, Indiana Univ. Math. J. 29 (1980), no. 5, 639-681.

[5] J. Leray, Sur le mouvement d'un liquide visqueux emplissant l'espace, Acta Math. 63 (1934), no. 1, $193-248$.

[6] P. Maremonti, A note on Prodi-Serrin conditions for the regularity of a weak solution to the Navier-Stokes equations, J. Math. Fluid Mech. 20 (2018), no. 2, 379-392.

[7] T. Miyakawa and H. Sohr, On energy inequality, smoothness and large time behavior in $L^{2}$ for weak solutions of the Navier-Stokes equations in exterior domains, Math. Z. 199 (1988), no. 4, 455-478.

[8] G. Prodi, Teoremi di tipo locale per il sistema di Navier-Stokes e stabilitådelle soluzioni stazionarie, Rend. del Sem. Matem della Univ. di Padova, 32 (1962), 173-182. 